\title{
ON THE IRRELEVANCE OF TRANSFORMATIONAL GRAMMAR TO SECOND LANGUAGE PEDAGOGY
}

\author{
John T. Lamendella \\ The University of Michigan
}

\begin{abstract}
Many scholars view transformational grammar as an attempt to represent the structure of linguistic knowledge in the mind and seek to apply transformational descriptions of languages to the development of second language teaching materials. It will be claimed in this paper that it is a mistake to look to transformational grammar or any other theory of linguistic description to provide the theoretical basis for either second language pedagogy or a theory of language acquisition. One may well wish to describe the abstract or logical structure of a language by constructing a transformational grammar which generates the set of sentences identified with that language. However, this attempt should not be confused with an attempt to understand the cognitive structures and processes involved in knowing or using a language. It is a cognitive theory of language within the field of psycholinguistics rather than a theory of linguistic description which should underlie language teaching materials.
\end{abstract}

A great deal of effort has been expended in the attempt to demonstrate the potential contributions of the field of descriptive linguistics to the teaching of second languages and, since the theory of transformational grammar has become the dominant theory in the field of linguistics, it is not surprising that applied linguists have sought to apply transformational grammar to gain new insights into the teaching of second languages. It will be claimed in this paper that it is a mistake to look to transformational grammar or any other theory of linguistic description to provide the theoretical basis for either second language pedagogy or a theory of language acquisition. That is, what is needed in the field of language teaching are not applied linguists but rather applied psychologists.

The primary goal of the language teacher is to instill in the student abilities in the production and comprehension of the target language which are comparable to those of the native speaker. Before adequate methods of language pedagogy can be developed, textbook writers and teachers will have to have access to a theory 
of human language acquisition and an understanding of the psychological representation of linguistic knowledge in the mind. Anything less puts the teacher in the position of merely presenting data to the student in a hit and miss fashion with no principled basis for deciding what it is that should be taught, the order of presentation, or how to give adequate explanations. Many scholars view transformational grammar as an attempt to represent the structure of linguistic knowledge in the mind and seek to apply transformational descriptions of languages directly to the development of second language teaching materials. If it is true that transformational grammar is a cognitive theory of language which is intended to make claims regarding the psychological representation of knowledge, then applied linguists who base their teaching materials on transformational grammar may feel that they are on the right track. If, however, transformational grammar makes no psychological claims, then just what is its status and what does it have to say to the language teacher?

When we consider people's ability to acquire, store, and use language and ask what kind of theory would "explain" or "characterize" human linguistic competence, we realize that there are at least three approaches open to us. These three approaches correspond to three "levels" of theories which may be constructed to describe human behavior. First, we may be concerned with a neurophysiological, biochemical description of the actual physical processes involved in language. Meaningful statements on this level are certainly far off in the future and it is not obvious that neurophysiology will contribute anything to the language teacher. Secondly, our goals may be in the field of descriptive linguistics and lead us to formal descriptions of languages and a theory of linguistic description. Thirdly, we may be concerned with developing a cognitive theory of language within the field of psycholinguistics. It is only the latter type of theory which could be utilized in the development of a theory of language acquisition. This is true because theories of linguistic description are concerned with the abstract or logical structure of languages and consider a language as an autonomous system isolated from the psychological processes which result in the acquisition, storage, and use of linguistic knowledge. Theories of linguistic description claim nothing regarding the way in which the knowledge of language processes and data is structured and stored in the mind (what might be called a theory of linguistic competence) or how a person acquires and utilizes these cognitive structures in producing or understanding sentences (that is, linguistic performance). Both theories of competence and theories of performance as defined here are beyond 
the goals of descriptive linguistics if we restrict these goals to the description of the abstract structure of linguistic utterances. No attempt is made to integrate theories of linguistic description with the concerns of psychologists interested in constructing models of other cognitive processes such as problem solving, perception, long and short-term memory, concepts and concept formation. Of course, descriptive linguistics has validity as a field of scientific endeavor but it should not be confused with the attempt to understand human language as a psychological phenomenon. The viewpoint of a cognitive theory of language must be psychological, that is, it should be concerned with describing people rather than describing languages. In addition, there must be at least some indication of how such a theory might be integrated with other cognitive functions and the models which have been proposed to explain them.

Recently, attempts have been made to formalize linguistic descriptions and to develop theoretical frameworks within which languages could be described. Unfortunately, at the present time there exists confusion in the field regarding the status of theories which have been proposed. In particular, the theory of transformational grammar proposed primarily by Chomsky and deriving in part from the work of Harris, although in origin a descriptive theory having nothing to do with human cognitive processes, is seen by some as having great relevance to a cognitive theory of human language.

This confusion could perhaps be lessened if we made a firm distinction between the goals, implications, and status of the theory itself and statements which transformationalists have made concerning their theory of description as well as their conjectures regarding the structure of language in the mind. Consider the following quotations from Chomsky:

(i) "The person who has learned a language has internalized a system of rules that relate sound and meaning in a particular way... The linguist, constructing a grammar of a language, is, in effect, proposing a hypothesis concerning this internalized system" (1968: 23)

(ii) "The problem for the linguist, as well as for the child learning the language, is to determine from the data of performance the underlying system of rules that has been mastered by the speakerer-hearer and that he puts to use in actual performance. Hence, in the technical sense, linguistic theory is mentalistic, since it is concerned with discovering a mental reality underlying actual behavior." (1965:4)

We may agree with Chomsky when he says that people who have learned a language possess something that may be called a 
system of rules, but we must disagree with him if he means to imply that the linguist's grammar is necessarily a hypothesis concerning the structure of this system of rules. If the grammar that Chomsky refers to is a transformational grammar, then it is not at all clear that one could consider a grammar a theory of how knowledge of a language system is structured in the mind. If a transformational grammar is intended to be a cognitive theory, not only may it be rejected out of hand as totally inadequate, but it will be argued that, in principle, no refinement of the theory could make it an adequate theory of the kind needed.

Chomsky would not claim (although some have) that the rules of a transformational grammar are intended to be isomorphic with the cognitive system of 'rules' that people possess after having learned a language. The rules and categories of a transformational grammar are part of an attempt to describe the set of sentences which constitute a "language." We may feel that by providing structural descriptions of sentences we have 'characterized' the cognitive structures stored by the speaker-hearer, but this use of the term "characterize" should not lead us to believe that we have therefore discovered or even attempted to discover the mental reality which underlies linguistic behavior.

Structural linguists have also attempted to describe the grammatical structure of languages, giving the constituent structure of the sentence patterns in the language. Neither they themselves nor anyone else was ever tempted to consider their grammars to be hypotheses concerning the speaker-hearer's internal linguistic knowledge. A transformational grammar may provide a better description in that it attempts to be explicit and considers a wider range of language data, but both types of grammars attempt to describe language structure. We cannot say that a transformational grammar makes cognitive claims while a structuralist grammar does not.

One might feel that because it has a level of "deep structure" which structuralist formulations lacked, transformational grammar alone may be considered a hypothesis concerning the knowledge structure of a speaker-hearer, but in a transformational grammar, the level of deep structure is an artifact of the system of rewrite rules and should by no means be equated with an attempt to describe the cognitive structures which underlie sentences in language use. Some psycholinguists (e.g., McNeill 1966), after concluding that people must have knowledge of language structure stored and some mechanism for mapping one kind of structure into another, wish to equate the cognitive structures with deep structure syntactic phrase markers and the mapping operation with the transforma- 
tional rules found in a descriptive grammar. Notice, however, that transformations as defined by Chomsky are not processes (psychological or otherwise) but are merely statements of the well-formedness conditions which hold between two tree structures. Phrase structure rules "generate" trees only in the sense that they define the structure of trees. They do not imply a cognitive system of phrase structure rules which "produce" trees. There is no empirical evidence that human beings possess deep syntactic phrase markers as part of their linguistic knowledge or that people go through a process of categorial decomposition of the $S \rightarrow N P+V P$ variety. All that can be stated is that in Chomsky's theory of linguistic description there is a level of deep structure trees, defined by phrase structure rules, which map onto surface structure trees.

A further reason for rejecting the direct transfer of Chomsky's theory of linguistic description to discussions of cognitive structures is that the tree structures in Chomsky's theory are inherently incapable of formally representing the structure of linguistic knowledge in the mind. A base categorial component such as Chomsky's is perhaps powerful enough to generate symbols in a correct order with the correct constituent structure, and it is therefore not $a$ priori impossible that it could serve as the basis for a sentence generator in a theory of linguistic description. If, however, our concern is with a cognitive theory of language, then it is demonstrably inadequate. Trees are only one form of graph structure and not particularly powerful when compared to other more complex graphs used to model psychological processes.

Since most of our technical vocabulary is used both in the descriptive theory where it receives formal definition and in our conjectures regarding cognitive structures, it is not surprising that many people are willing to believe that the framework of transformational grammar is suitably extended to a concern for a cognitive theory of language. The term "generative grammar" is used to mean both the formal device constructed by a linguist and the hypothetical cognitive system which enables people to engage in linguistic behavior. A linguist writes "rules" and people are said to have "internalized" a system of "rules."

A further example of the desire to give cognitive status to the theory of transformational grammar is seen in the following quotation from Chomsky. (1968), pp. 4-5:

\footnotetext{
"Knowledge of a language involves the ability to assign deep and surface structures to an infinite range of sentences, to relate these structures appropriately, and to assign a phonetic interpretation to the surface structure and to construct a semantic interpretation on the basis of the grammatical relations of the deep structure."
} 
One isn't too surprised to discover that these abilities correspond to the operations and components of a transformational grammar. If we accepted this list of abilities as in fact what is involved in possessing "knowledge of a language," we might be tempted to consider transformational grammar as a proposal for a theory of human language. However, when we look for the cognitive and behavioral counterparts to these four abilities, it is not clear what could be intended, especially when we bear in mind that these abilities are meant to reflect the structure of stored knowledge or "competence."

It has been primarily due to discussions of the compentenceperformance distinction and statements to the effect that a transformational grammar "characterizes the knowledge of the ideal speaker-hearer" that some linguists and psycholinguists are willing to believe that a transformational grammar is concerned with describing the cognitive structures which give rise to human language. The distinction between a theory of competence (i.e., a grammar) and a theory of performance has served to give transformational grammar an aura of cognitive significance while allowing it to ignore all questions of cognitive structure and processes.

At issue here are general assumptions concerning the conditions under which a formal model may be assumed to provide a description of unobservable systems of cognitive structures. Identity in terms of empirical consequences is not sufficient to insure that a model is in fact a description of the operations and structures of an unobservable cognitive system. The output of an electronic calculator may be empirically indistinguishable from the output of an ideal human adder-subtractor (e.g., 4,564 given the instruction to find the produce of $326 \cdot 14$ ), yet few would wish to claim that a circuit diagram for a calculator is necessarily a description of the way in which knowledge of arithmetic operations is represented in the mind.

Consider the schema presented in figure 1 which represents the components and input-output relations of a transformational grammar as formulated by Chomsky. If a transformational grammar for a language existed, it would assign deep and surface structures to an infinite range of sentences in the sense that the base component would generate deep structure phrase markers and the transformational component would map these onto surface structures. Structures generated by the grammar would be related appropriately if empirical evidence in the form of native speaker judgments of relatedness was reflected by phrase markers sharing appropriate elements of structure. How the phonological component of a transformational grammar could assign a phonetic 
figure 1

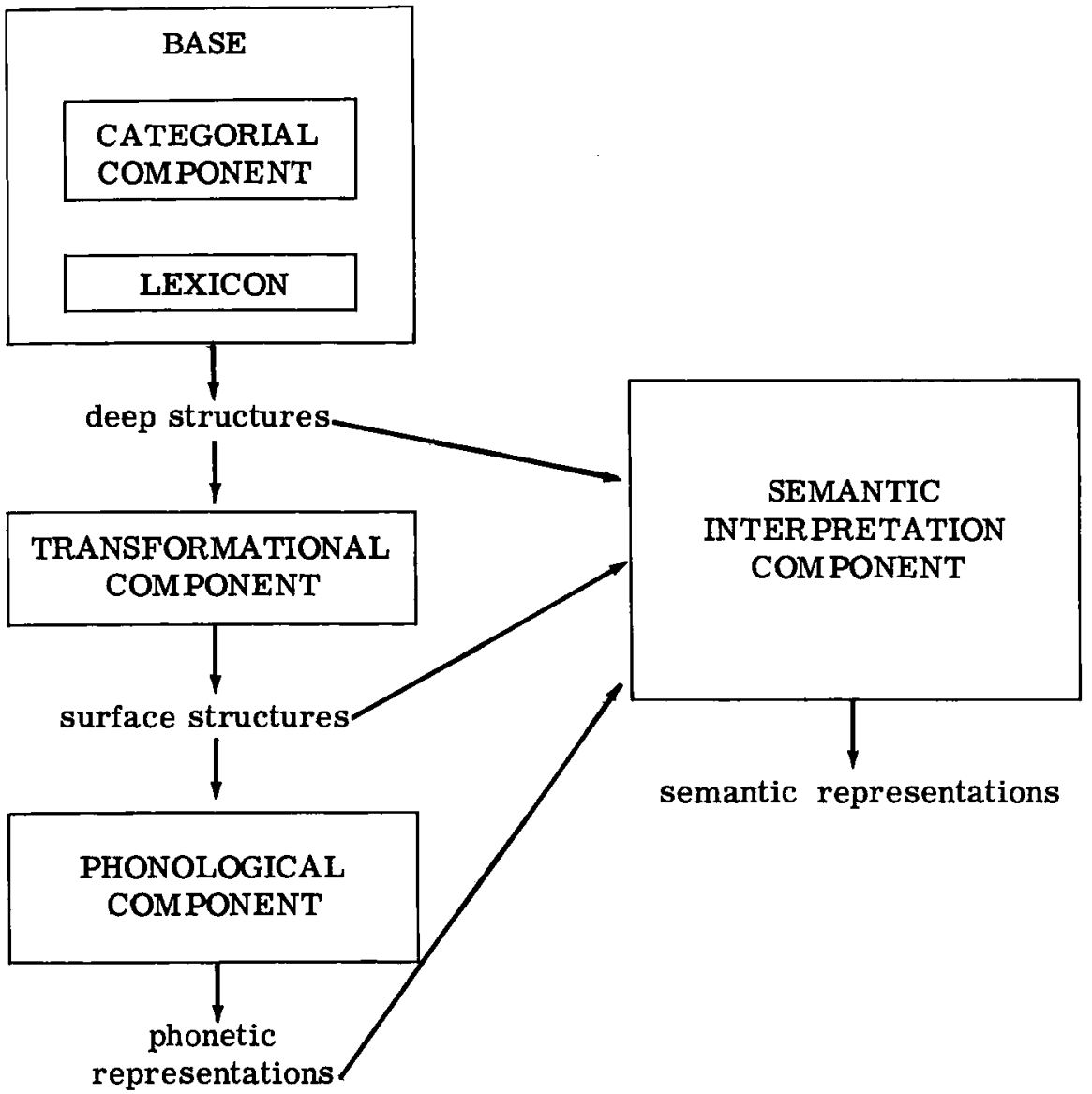

representation given an input of surface structure phrase markers with lexical items and grammatical formatives as terminal nodes, has been outlined in Chomsky and Halle (1968). The operation and content of the semantic interpretation component is almost completely unspecified.

Taken literally as a hypothesis concerning the internal representation of linguistic knowledge which people possess and use to produce and understand language, this schema would seem to imply that people first run through their categorial component and select a grammatical structure, insert permissible lexical items, and then decide what they want to say. As noted in Chomsky (1969), this is certainly not what goes on in language production and the organi- 
zation of a transformational grammar is not intended to make claims regarding the temporal or logical order of psychological processes relevant to language performance. It is meaningless to talk of an intrinsic order of a mapping operation such as the mapping between deep and surface structures. Also, in a theory of linguistic description such as transformational grammar, it makes no sense to posit a necessary order for the selection of structures. Chomsky's grammar generates quadruples (a deep structure, a surface structure, a phonetic representation, and a semantic representation). It cannot be said that one type of structure must be selected before or after another.

The result of the lack of implicit order of operations, however, is the conclusion that the schema of figure 1 can make no cognitive claims on the gross level of componential structure. In any hypothesis concerning the structure of knowledge in the mind, both the chronological and logical order of psychological processes must be implicit if it is this knowledge which is relevant to language production and comprehension. Moreover, it is probably an oversimplification to assume that a transformational grammar can be made relevant to a theory of performance merely by superimposing a preferred order of operations. There is evidence that language production and comprehension cannot simply be a matter of applying the unique grammar developed in a 'theory of competence': e.g., this would imply that it is easier to generate new sentences than to understand new sentences but it is generally recognized that people (and children in particular) understand many more sentences than they can produce; also, the fact that people understand many different dialects shows that more than the sentence production grammar must be available in comprehension. (These observations are from Quillian 1966)

It is probably true that we must first have a theory of how people's knowledge of language is structured and stored in the mind before we can develop a theory of how this knowledge is used, but a transformational grammar is not the description of this knowledge that we require.

One could perhaps counter this assertion with the claim that the constituent structures posited in a linguistic description do correspond to functioning psychological units. People have strong intuitions regarding the units of sentence structure and it seems possible to corroborate experimentally the claims which a grammar makes in this regard. (See, for example, Fodor \& Bever 1965) When linguists describe languages they do attempt to accommodate the judgments of native speakers concerning the constituent parts of sentences. 
The techniques of Immediate Constituent Analysis developed in structural linguistics were part of an attempt to describe the constituent structure of sentences. (See, for example, Wells 1947) Transformational grammar has attempted to overcome the difficulties inherent in any attempt to describe language structure by merely slicing up sentences. IC analysis was not equipped to deal with such things as discontinuous constituents, some types of structural ambiguity, and people's intuitions regarding the relationships between sentences. A transformational grammar can provide an account of how discontinuous elements in a sentence belong to the same constituent; it can disambiguate identical surface structures; and it does implicitly express relationships between sentences. However, the relevant question is whether the apparatus employed in a transformational grammar to accomplish these descriptive goals bears any relationship to psychological processes which function in human language.

Consider the following sentences which are examples of one type of "structural ambiguity":

(11a) John is easy to please.

(11b) John is eager to please.

In a transformational grammar they might be assigned superficial phrase markers of the following sort:

(11a)' and (11b)'

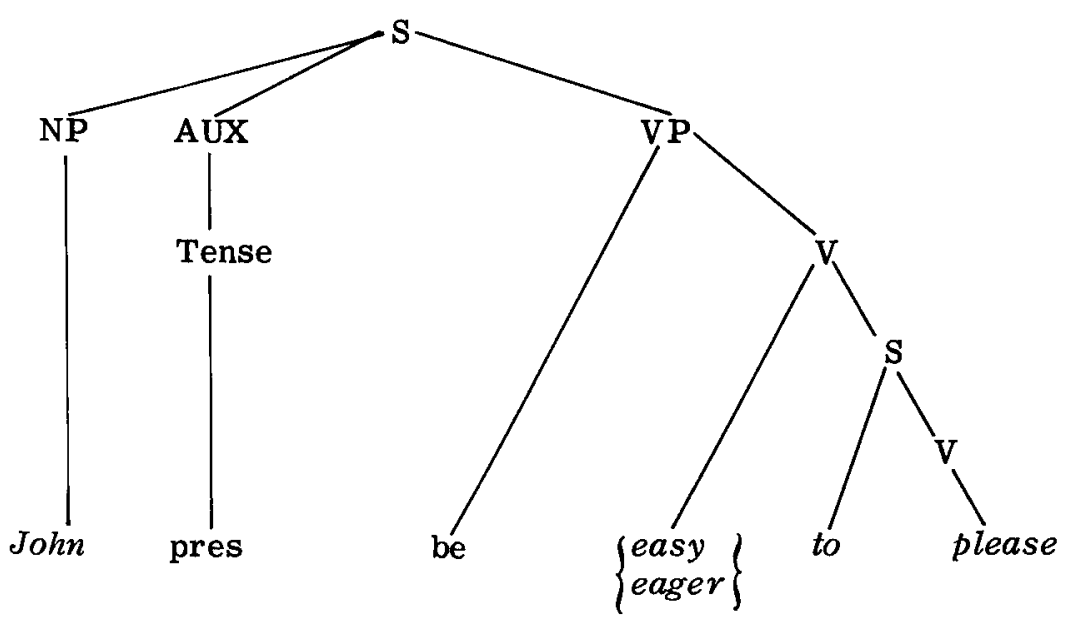


Note that while the surface structures (11a)' and (11b)' have exactly the same constituent structure and the same labels on nodes, they are in fact understood by people to be very different in the relationships between their parts. John is, in some sense, the object of please in (11a) but the subject of please in (11b). The sentences differ systematically in their relationships to other sentences which are paraphrases and/or expansions of (11a) and (11b). Compare,

(12) It is easy for Mary to please John.

(13) *It is eager for Mary to please John.

(14) John is eager to please Mary.

(15) *John is easy to please Mary.

On the basis of such evidence, a transformational grammar would claim that the two sentences, although possessing identical surface structures, differ in that they must be derived from different underlying syntactic structures (i.e., different K-initial Phrase Markers).

Following these criteria we may conclude that the following pair of sentences also exhibit the same type of structural ambiguity.

(16a) John shot Mary.

(16b) John hated Mary.

Once again two sentences have the same constituent structure and the same labelling. They differ in the relations between their parts and in their relationships to other sentences in a way that is parallel to (11a) and (11b), i.e.,

(18) *John hated Mary with a gun.

(19) John had hatred for Mary.

(20) *John had shooting for Mary.

It seems not to be the case, however, that (16a) and (16b) would be derived from different underlying syntactic structures in a transformational grammar of the type proposed by Chomsky except insofar as the lexical entries for the verbs shoot and hate would differ. Moreover, transformational grammar provides no formal way to distinguish the cases such as (11a) and (11b) where different deep structures are posited and cases such as (16a) and (16b), where we would feel uneasy in doing so. 
Empirical data which can be gathered concerning such sentences as (11a)-(11b) and (16a)-(16b) show that the pairs of sentences are understood differently and that they would have different semantic representations. There is, however, no evidence that it would be necessary or desirable to posit different syntactic structures from which they must be derived or that people go through a process of categorial decomposition of the $S \rightarrow \mathrm{NP}+\mathrm{VP}$ type, resulting in different deep syntactic structures for, e.g., (11a) and (11b).

Constituents which have been determined to exist at the level of occurring sentences together with the words which label them, are generally taken to define syntactic categories. Within transformational grammar, syntactic categories such as $S$ and NP receive formal definition by virtue of their position in an ordered set of rewrite rules which define the supercategories and subcategories of a particular category. What is not obvious is that these categories should be considered to function cognitively in a way analogous to their role in the phrase structure rules of the base component of a transformational grammar. Deep syntactic categories are not typically posited after careful investigation into the categories which people might actually possess, but amount to those categories which seem to be necessary if our goal is to construct a device which will strongly generate the set of sentences which, for Chomsky, constitutes the language. Instead of employing the symbols $S$ and NP, one might as well use $\$$ and $c$ as category labels in a transformational grammar. However, using the words "sentence" and "noun phrase" carry over into the notation certain connotations of significance that $\$$ and $\$$ lack.

Transformational theory is an extension and a formalization of the concerns of structural linguists. The goals of the theory of transformational grammar should be taken to be those originally expressed by Chomsky:

"The fundamental aim in the linguistic analysis of a language $L$ is to separate the grammatical sequences which are the sentences of $L$ from the ungrammatical sequences which are not sentences of $L$ and to study the structure of the grammatical sequences. The grammar of $\mathrm{L}$ will thus be a device that generates all the grammatical sequences of $\mathrm{L}$ and none of the ungrammatical ones." $(1957: 13)$

More recent discussions imputing cognitive status to the theory should be taken as a verbal overlay which has not been matched by any substantive change in either the goals or the status of the theory itself.

Only a short time after transformational grammar became generally accepted as the reigning theory in the field of linguistics, and in addition, was voted the theory most likely to succeed in 
providing an account of language which could be utilized in other scientific disciplines such as psychology, machine translation, and high school English teaching, dissention and heresy drove a firm wedge into the once solid ranks of transformationalists. There are now at least two different theories of transformational grammar: the standard theory of the "lexicalists," represented by Chomsky, Jackendoff, etc. and the revisionist theory of the "transformationalists" (henceforth "neo-transformationalists"), represented by McCawley, Lakoff, Ross, etc.

The neo-transformationalists have rejected Chomsky's standard theory as inadequate to represent the facts of language. Chomsky, on the other hand, has dismissed the proposals of the neo-transformationalists as inferior "notational variants" of his theory. The schism among transformationalist grammarians has had its origin in attempts to describe language structure within the framework of Chomsky's theory. For some linguists, it seemed necessary to posit increasingly more abstract deep structures than those which had been proposed in Chomsky (1965). This increased degree of abstractness appeared necessary if the grammar were to generate all acceptable surface structures, block unacceptable surface structures, and account for the unacceptability of certain sentences. Also, the evaluative criterion of simplicity so prominent in earlier transformational grammar gave way to a greater concern for a criterion of naturalness. Thus, there was dissatisfaction with categories and structures which, while adequate to generate correct strings, were intuitively felt not to reflect the facts of human language. Moreover, if substantive linguistic universals were to play any role in grammar, deep structures had necessarily to become less language specific.

The deep structures which were deemed most natural and correct became further and further removed from the superficial form of sentences. An attempt was made to justify the inclusion of abstract elements and embedded sentences on syntactic grounds. However, the deep syntactic structures posited for sentences became more and more to suspiciously resemble exactly the kind of information one would expect to find in the semantic representation of sentences. Also, transformational rules which operated on these structures came to look like semantic interpretation rules in reverse. These considerations interacted with many other observations and problems which had resulted from attempting to work within transformational grammar and the result was the hypothesis that it was neither necessary nor desirable that a grammar should have both a syntactic categorial component which generates syntactic phrase markers and a semantic interpretation component which 
derives semantic representations from syntactic structures. What was proposed was a single component which would directly generate semantic representations expressing meaning and containing sufficient information for the operation of transformations. It is in this sense that the term "generative semantics" has been used and it is in this sense that the theory of the neo-transformationalists is semantically based.

In place of the organization of the standard theory of transformational grammar represented in figure 1, the statements of the neo-transformationalists can be taken to imply a grammar having the componential structure given in figure 2.

Even if it were true that the theory proposed by the neo-transformationalists is a mere notational variant of Chomsky's standard theory (and it is not), these linguists have in fact taken up a greater concern with investigating the nature of human language and deemphasized the goal of mechanically generating strings with the correct order and the appropriate constituent structure. However, their goals still lie within the field of descriptive linguistics and it is not possible to see their theory as having direct relevance for a cognitive theory of language.

Since one has the feeling that meaning is what language is all about, any theory of human language would hopefully consider a theory of meaning as being of prime importance. Given the actual goals (as against the expressed goals) of Chomsky's standard theory, it is not surprising that the aspect of human language which has been given the least consideration is that of meaning and semantic representation. While the neo-transformationalists place major emphasis on semantic representations as underlying surface syntactic structures, their concern for meaning has been purely from the point of view of descriptive linguistics. Meaning, however, is not a function of words and sentences as phonological or grammatical entities, but of the intention of the speaker and the ability of the hearer to reconstruct this intended meaning. One implication of this statement which has been ignored by most linguists, philosophers, and (sad to say) psychologists is that if we are to discuss meaning and semantic representation with cognitive import, it must be discussed in the context of a theory which attempts to explain human thought processes. In the same sense in which one cannot describe syntax in the abstract and hope to achieve a model of how syntactic structures are stored in the mind, one cannot isolate a "linguistic" meaning for sentences which is describable apart from a concern with cognitive structures and expect this linguistic meaning to reflect the actual representation of meaning in the mind. A theory of semantics must be part of a theory of human 
figure 2

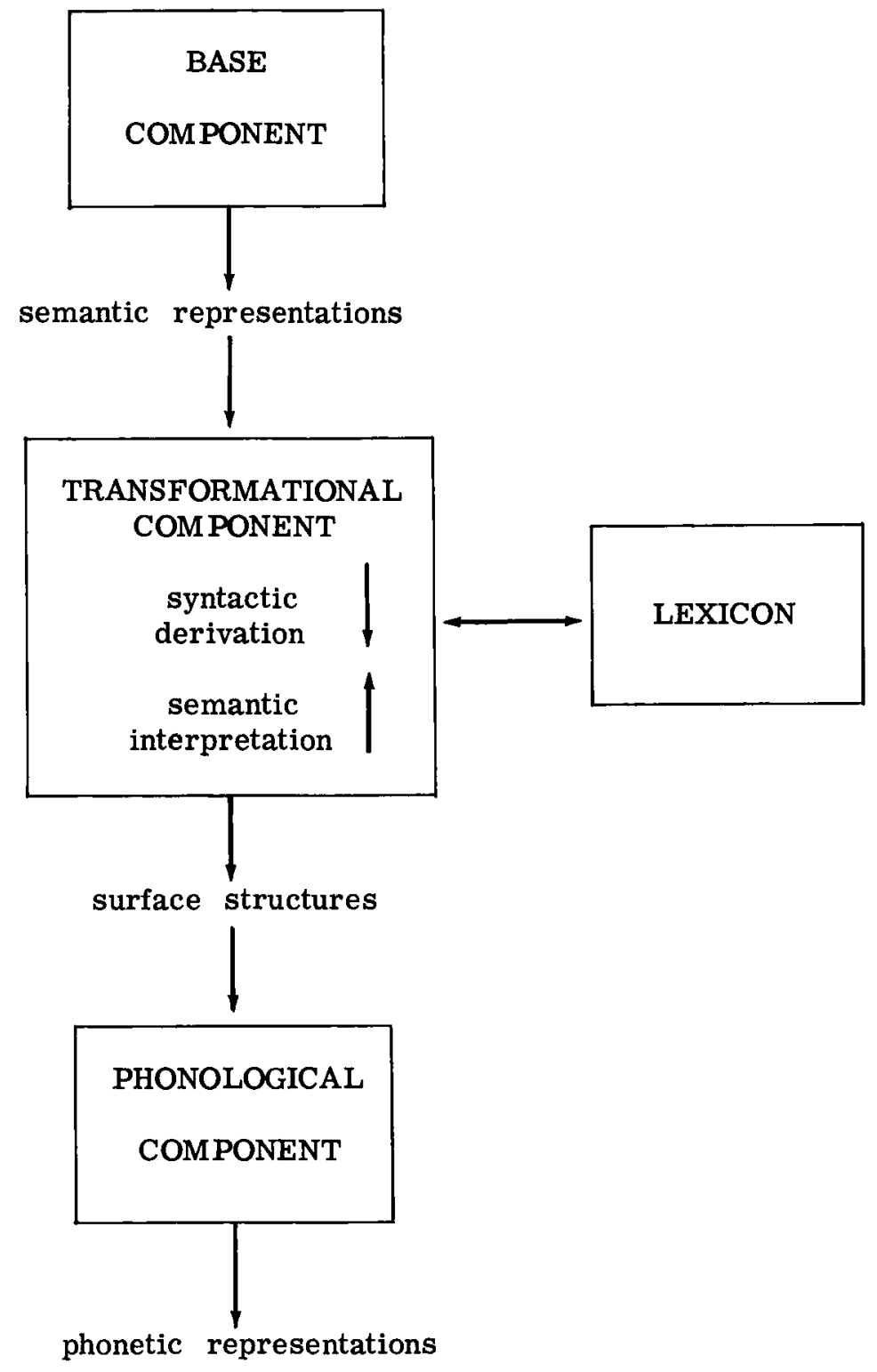


cognition if it is to have any relevance to a cognitive theory of human language and, in addition, the cognitive representation of semantic information underlying sentences may clearly be equated with the little understood cognitive entities called "thoughts" or "ideas."

The fact that there are people who have knowledge of the world, solve problems, perceive, categorize events, remember, create (in short, people who think) but who have not acquired any language at all (see, e.g., Lenneberg 1967) is sufficient to show that thought (and therefore meaning) must be distinct from language and capable of description apart from any theory of language. The converse, however, is not true; that is, any cognitive theory of language is dependent on a general theory of human cognition. If we equate a concern for semantics with a concern for the cognitive processes which originate thought, then a theory of semantics must be taken to be separate from (although related to) a theory of grammar.

The process of producing thoughts and encoding these thoughts into language necessarily involves the retrieval of information which is stored in memory. The initial goal of a cognitive theory of semantics, therefore, should be the development of hypotheses concerning the structure of the knowledge which people have stored about the world and the way in which information is retrieved from memory structures. That is, it is precisely long-term memory structures which should form a major part of the semantic component in a cognitive theory of language. (See, e.g., Lamendella 1969a) The psycholinguist may well be concerned with an account of how, given a thought or idea retrieved or produced from memory structures, a speaker encodes this thought into language and utters a sentence; and how, conversely, a hearer decodes sentences and attempts to reconstruct the thought which was in the mind of the speaker. However, it is the cognitive psychologist who is concerned with developing the theory of cognitive processes and the representations of ideas which the psycholinguist may use.

The descriptive linguist, of course, may work toward the development of a theory of linguistic description and the description of particular languages; including an account of semantic representations which is divorced from any psychological considerations. However, the status of the theories which linguists develop should be made quite clear. In spite of the claims which Chomsky has made about his theory seeking to give it cognitive status, neither the standard theory of transformational grammar nor the theory of generative semantics of the neo-transformationalists make hypotheses concerning the structure of linguistic knowledge in the mind. 
Chomsky and other transformational grammarians have contributed a great deal to our understanding of language and language pedagogy, for example, by pointing out the vacuity of behavioral psychology and the uses to which it has been put in language teaching. However, an examination of recent second language textbooks shows just how little of any consequence has been contributed by the theory of transformational grammar itself to the development of teaching materials. Once we get beyond the superficial misapplications of terminology (e.g., talking about "two surface structures being derived by transformational rule from a common deep structure" when it is merely meant that two sentences are related in meaning), we see that the formal structures and categories defined in a transformational grammar have not been put to pedagogical advantage in second language teaching (Cf. Lamendella 1969b). Moreover, theories of linguistic description are relevant to language teaching only to the extent that they form part of the data which psycholinguists may use in constructing a cognitive theory of language. It is this theory which may properly be utilized as the theoretical basis for second language pedagogy.

\section{REFERENCES}

Chomsky, Noam. 1957. Syntactic Structures. The Hague: Mouton.

Chomsky, Noam. 1965. Aspects of the Theory of Syntax. Cambridge: M.I.T. Press.

Chomsky, Noam. 1968. Language and Mind. New York: Harcourt, Brace, and World, Inc.

Chomsky, Noam. 1969. Deep Structure, Surface Structure, and Semantic Interpretation. Ditto.

Chomsky, Noam and Morris Halle. 1968. The Sound Pattern of English. New York: Harper and Row.

Fodor, Jerry A. and Thomas G. Bever. 1965. Psychological Reality of Linguistic Segments. Journal of Verbal Behavior 4.414-20.

Lamendella, John T. 1969a. Long-Term Memory, Conceptual Structure, and a Theory of Generative Semantics. Phd. Dissertation, University of Michigan.

Lamendella, John T. 1969b. Review of Modern English, by W. E. Rutherford. Language Learning 19.1\&2.147-160.

Lenneberg, Eric. 1967. Biological Foundations of Language. New York: Wiley and Sons.

McNeill, David. 1966. Developmental Psycholinguistics. The Gensis of Language: A Psycholinguistic Approach, ed. by F. Smith and G. Miller, 15-84. Cambridge: M.I.T. Press.

Quillian, Ross. 1966. Semantic Memory. Report AFCRL-66-189. Cambridge, Massachusetts: Bolt, Beranek, and Newman.

Wells, Rulon. 1947. Immediate Constituents. Language 23.2. 